\title{
Evolution and Dynamics of Cusped Light-Like Wilson Loops in Loop Space
}

\author{
F.F. Van der Veken*, I.O. Cherednikov" ${ }^{* \dagger}$ and T. Mertens* \\ * Departement Fysica, Universiteit Antwerpen, B-2020 Antwerpen, Belgium \\ ${ }^{\dagger}$ BLTP JINR, RU-141980 Dubna, Russia
}

\begin{abstract}
We discuss the possible relation between the singular structure of TMDs on the lightcone and the geometrical behaviour of rectangular Wilson loops.
\end{abstract}

Keywords: Wilson loops, loop space, Makeenko-Migdal equations, non-perturbative techniques, renormalization, large $N_{c}$-expansion, gauge/string duality, evolution of TMDs

PACS: 11.10.Gh,11.15.Pg,11.15.Tk,11.25.Tq

\section{INTRODUCTION}

Transverse momentum dependent parton density functions (or TMDs for short) are known to have a more complex singularity structure than collinear parton density functions. Common singularities like ultraviolet poles can be removed by general methods like standard renormalisation using the $R$-operation. In the case of a light-like TMD however, where at least one of its segments is on the light-cone, it is not entirely clear whether standard renormalisation remains a sufficient technique, due to the emergence of extra overlapping divergences. A standard TMD can be defined as [1]:

$$
f\left(x, \boldsymbol{k}_{\perp}\right)=\left.\frac{1}{2} \int \frac{\mathrm{d} z^{-} \mathrm{d}^{2} z_{\perp}}{2 \pi(2 \pi)^{2}} e^{i k \cdot z}\left\langle P, S\left|\bar{\psi}(z) U^{\dagger}(z ; \infty) \gamma^{+} U(\infty ; 0) \psi(0)\right| P, S\right\rangle\right|_{z^{+}=0}
$$

where the Wilson lines are split into their longitudinal and transversal parts:

$$
\begin{aligned}
U(\infty, 0) & =U\left(\infty^{-}, \infty_{\perp} ; \infty^{-}, \mathbf{0}_{\perp}\right) U\left(\infty^{-}, \mathbf{0}_{\perp} ; 0^{-}, \mathbf{0}_{\perp}\right) \\
& =\mathscr{P} e^{-\mathrm{i} g \int_{0}^{\infty} \mathrm{d} z_{\perp} A_{\perp}\left(\infty^{-}, z_{\perp}\right)} \mathscr{P} e^{-\mathrm{i} g \int_{0}^{\infty} \mathrm{d} z^{-} A^{+}\left(z^{-}, \mathbf{0}_{\perp}\right)}
\end{aligned}
$$

When on light-cone, this TMD will posses extra divergences proportional to $\frac{1}{\varepsilon^{2}}$ (when using dimensional regularisation). These will give the only contribution to the evolution equations, governed by the cusp anomalous dimension $[2,3]$

$$
\Gamma_{\text {cusp }}=\frac{\alpha_{s} C_{F}}{\pi}(\chi \operatorname{coth} \chi-1) \quad \stackrel{\text { on-LC }}{\longrightarrow} \frac{\alpha_{s} C_{F}}{\pi}
$$

where $\chi$ is the cusp angle (in literature sometimes referred to as a 'hidden cusp') which goes to infinity in the light-cone limit. In the next sections, we will show that a specific type of Wilson loop, namely rectangular loops with light-like segments on the null-plane, has its singularity structure analogous to on-LC TMDs, which feeds the idea that there might exist a duality between those two objects. 


\section{WILSON LOOPS AS ELEMENTARY OBJECTS IN LOOP SPACE}

A general Wilson loop is defined as

$$
\mathscr{W}[C]=\frac{1}{N_{c}} \operatorname{tr}\left\langle 0\left|\mathscr{P} e^{\mathrm{i} g \oint_{C} \mathrm{~d} z^{\mu} A_{\mu}^{a}(z) t_{a}}\right| 0\right\rangle
$$

where $C$ is any closed path and $A_{\mu}$ is taken in the fundamental representation. This loop is a pure phase, traced over Dirac indices and evaluated in the ground state, transforming coordinate dependence into path dependence. As is known (see [4, 5]), Wilson loops can be used as elementary objects to completely recast QCD in loop space. To achieve this, the definition of a Wilson loop needs to be extended to make it dependent on multiple contours:

$$
\mathscr{W}_{n}\left(C_{1}, \ldots, C_{n}\right)=\left\langle 0\left|\Phi\left(C_{1}\right) \ldots \Phi\left(C_{n}\right)\right| 0\right\rangle \quad \Phi(C)=\frac{1}{N_{c}} \operatorname{tr} \mathscr{P} e^{\mathrm{i} g \oint_{C} \mathrm{~d} z^{\mu} A_{\mu}(z)}
$$

All gauge kinematics are encoded in a $\mathscr{W}_{1}$ loop, and all gauge dynamics are governed by a set of geometrical evolution equations, the Makeenko-Migdal equations [6]:

$$
\partial^{v} \frac{\delta}{\delta \sigma_{\mu v}(x)} \mathscr{W}_{1}(C)=g^{2} N_{c} \oint_{C} \mathrm{~d} z^{\mu} \delta^{(4)}(x-z) \mathscr{W}_{2}\left(C_{x z} C_{z x}\right)
$$

where two (geometrical) operations are introduced, namely the path derivative $\partial_{\mu}$ and

the area derivative $\frac{\delta}{\delta \sigma_{\mu v}(x)}$ [6]. Although the Makeenko-Migdal equations provide an elegant method to describe the evolution of a generalised Wilson loop solely in function of its path, they have their limitations. For starters, they are not closed since the evolution of $\mathscr{W}_{1}$ depends on $\mathscr{W}_{2}$. Formally, this limitation is superfluous in the large $N_{c}$ limit since then we can make use of the 't Hooft factorisation property $\mathscr{W}_{2}\left(C_{1}, C_{2}\right) \approx \mathscr{W}_{1}\left(C_{1}\right) \mathscr{W}_{1}\left(C_{2}\right)$ [6], making the MM equations closed. The remaining limitations of the MM equations are more severe. For one, the evolution equations are derived by applying the SchwingerDyson methodology on the Mandelstam formula

$$
\frac{\delta}{\delta \sigma_{\mu v}(x)} \Phi(C)=\mathrm{i} g \operatorname{tr}\left\{F^{\mu v} \Phi\left(C_{x}\right)\right\}
$$

and using the Stokes' theorem. These might, as well as the area derivative, not be welldefined for all types of paths. In particular, all contours containing one or more cusps might induce some problematic behaviour, as it is (at least) not straightforward to define continuous area differentiation inside a cusp, nor it is to continuously deform a contour in a general topology [7]. This is somewhat bothersome, as most interesting dynamics lies in contours with cusps.

\section{EVOLUTION OF RECTANGULAR WILSON LOOPS}

Now we turn to a specific type of path, namely a rectangular path with light-like segments $\left(v_{i}^{2}=0\right)$ on the null-plane $\left(\boldsymbol{x}_{\perp}=0\right)$, as depicted in Figure 1. To investigate 


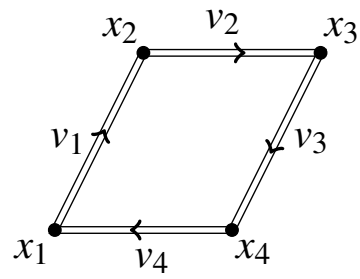

FIGURE 1. Parametrisation of a rectangular Wilson loop in coordinate space.

its singularity structure, we evaluate the loop (5) at one loop in coordinate space [8]:

$$
\mathscr{W}_{\text {L.O. }}=1-\frac{\alpha_{s} C_{F}}{\pi}\left(2 \pi \mu^{2}\right)^{\varepsilon} \Gamma(1-\varepsilon)\left[\frac{1}{\varepsilon^{2}}\left(-\frac{s}{2}\right)^{\varepsilon}+\frac{1}{\varepsilon^{2}}\left(-\frac{t}{2}\right)^{\varepsilon}-\frac{1}{2} \ln ^{2} \frac{s}{t}\right]
$$

where $s$ and $t$ are the Mandelstam energy/rapidity variables (note the positive sign in $t$ ):

$$
s=\left(v_{1}+v_{2}\right)^{2} \quad t=\left(v_{2}+v_{3}\right)^{2} \quad v_{i}=x_{i}-x_{i+1}
$$

Note the $\frac{1}{\varepsilon^{2}}$ poles, which are the overlapping divergences that stem from the light-like behaviour of the contour segments. The fact that they appear already at leading order renders this kind of Wilson loop non-renormalisable (at least not using the standard $R$-operation). The most straightforward way to manage them is by deriving an evolution equation for the loop. This is done by double differentiation (after rescaling $\left.\bar{s}=\pi e^{\gamma_{E}} \mu^{2} s\right)$ :

$$
\frac{\mathrm{d}}{\mathrm{d} \ln \mu} \frac{\mathrm{d}}{\mathrm{d} \ln s} \mathscr{W}_{\text {L.O }}=-2 \frac{\alpha_{S} C_{F}}{\pi}=-2 \Gamma_{\text {cusp }}
$$

where we recognise the cusp anomalous dimension in the light-cone limit from (4). Thus, as anticipated in the TMD case, the only contribution to the evolution equations stems from the overlapping divergences. Their concurrent appearance in the on-LC TMD case and in the case of an on-LC rectangular Wilson loop again hints to the existence of a duality between both.

\section{GEOMETRICAL BEHAVIOUR AND RELATION TO TMDS}

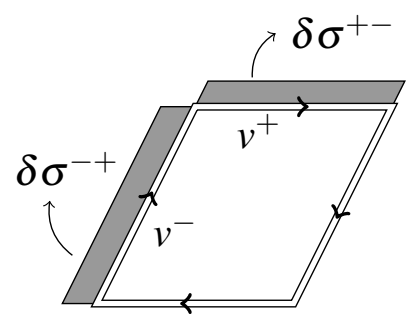

FIGURE 2. Angle-conserving deformations of a light-like rectangular loop on the null-plane.

In an attempt to combine the geometrical approach of the Makeenko-Migdal method with the evolution equations at leading order just derived, we investigate area dif- 
ferentiation on rectangular light-like loops on the null-plane, rendering the area differentials well-defined (see Figure 2) [7]. This gives $\delta \sigma^{+-}=\oint \mathrm{d} x^{-} x^{+}=v^{+} \delta v^{-}$and $\delta \sigma^{-+}=\oint \mathrm{d} x^{+} x^{-}=v^{-} \delta v^{+}$. Next we introduce the area variable $\Sigma$ :

$$
\Sigma \equiv v^{-} \cdot v^{+}=\frac{1}{2} s \quad \frac{\delta}{\delta \ln \Sigma}=\sigma_{\mu \nu} \frac{\delta}{\delta \sigma_{\mu v}}
$$

Replacing $s$ by $\Sigma$ in equation (11) gives $-4 \Gamma_{\text {cusp. }}$ Motivated by this, we conjecture a general evolution equation for light-like rectangular Wilson loops:

$$
\frac{\mathrm{d}}{\mathrm{d} \ln \mu}\left[\sigma_{\mu \nu} \frac{\delta}{\delta \sigma_{\mu \nu}} \ln \mathscr{W}\right]=-\sum_{i} \Gamma_{\mathrm{cusp}}
$$

Besides for light-like rectangular Wilson loops, equation (13) is expected to be valid for light-like TMDs, as they posses the same singularity structure. The area variable then gets replaced by the rapidity variable. This gives

$$
\frac{\mathrm{d}}{\mathrm{d} \ln \mu} \frac{\mathrm{d}}{\mathrm{d} \ln \theta} f\left(x, \boldsymbol{k}_{\perp}\right)=2 \Gamma_{\text {cusp }}
$$

The minus disappeared because $\theta \sim \Sigma^{-1}\left(\theta=\frac{\eta}{p \cdot v^{-}}\right.$and $p \sim v^{+}$, so $\left.\theta \sim\left(v^{+} v^{-}\right)^{-1}\right)$, and there is a factor 2 since we haven two (hidden) cusps. This result is very similar to the Collins-Soper evolution equations for off-LC TMDs.

\section{ACKNOWLEDGMENTS}

I'd like to thank the organisers of Diffraction 2012 for their hospitality and for the wonderful atmosphere during the conference.

\section{REFERENCES}

1. J. C. Collins and D. E. Soper, Nucl. Phys. B 193381 (1981); [Nucl. Phys. B 213545 (1983) Erratum]; J. C. Collins and D. E. Soper, Nucl. Phys. B 194445 (1982); A. V. Belitsky, X. Ji and F. Yuan, Nucl. Phys. B 656165 (2003); D. Boer, P. J. Mulders, F. Pijlman, Nucl. Phys. B 667201 (2003).

2. G. P. Korchemsky, A. V. Radyushkin, Nucl. Phys. B 2833421987.

3. I. O. Cherednikov and N. G. Stefanis, Phys. Rev. D 77094001 (2008); Nucl. Phys. B 802146 (2008); Phys. Rev. D 80054008 (2009); N. G. Stefanis and I. O. Cherednikov, Mod. Phys. Lett. A 242913 (2009).

4. S. Mandelstam, Phys. Rev. 1751580 (1968); K. G. Wilson, Phys. Rev. D 102445 (1974); Y. Nambu, Phys. Lett. B 80372 (1979); A. M. Polyakov, Phys. Lett. B 82247 (1979).

5. A. M. Polyakov, Nucl. Phys. B 164171 (1980); V. S. Dotsenko and S. N. Vergeles, Nucl. Phys. B 169 527 (1980); I. Ya. Arefeva, Phys. Lett. B 93347 (1980); N. S. Craigie and H. Dorn, Nucl. Phys. B 185 204 (1981); S. Aoyama, Nucl. Phys. B 194513 (1982); N. G. Stefanis, Nuovo Cim. A 83205 (1984). I. I. Balitsky and V. M. Braun, Nucl. Phys. B 311541 (1989).

6. Y. M. Makeenko and A. A. Migdal, Phys. Lett. B 88135 (1979) [Erratum-ibid. B 89437 (1980)]; Nucl. Phys. B 188269 (1981); A. A. Migdal, Annals Phys. 109365 (1977); Annals Phys. 126279 (1980); Phys. Rept. 102199 (1983).

7. I. O. Cherednikov, T. Mertens and F. F. Van der Veken, Phys. Rev. D 86085035 (2012).

8. I. A. Korchemskaya and G. P. Korchemsky, Phys. Lett. B 287169 (1992); A. Bassetto, I. A. Korchemskaya, G. P. Korchemsky and G. Nardelli, Nucl. Phys. B 40862 (1993). 
Copyright of AIP Conference Proceedings is the property of American Institute of Physics and its content may not be copied or emailed to multiple sites or posted to a listserv without the copyright holder's express written permission. However, users may print, download, or email articles for individual use. 